\title{
Well-posedness, wave breaking, Holder continuity and periodic peakons for a nonlocal sine- $\mu$-Camassa-Holm equation
}

\author{
Guoquan Qin ${ }^{1}$, Zhenya Yan $^{1}$, and Boling Guo ${ }^{2}$ \\ ${ }^{1}$ Academy of Mathematics and Systems Science Chinese Academy of Sciences \\ ${ }^{2}$ Institute of Applied Physics and Computational Mathematics
}

December 30, 2021

\begin{abstract}
In this paper, we investigate the initial value problem of a nonlocal sine-type $\mu$-Camassa-Holm $(\mu \mathrm{CH})$ equation, which is the $\mu$-version of the sine-type $\mathrm{CH}$ equation. We first discuss its local well-posedness in the framework of Besov spaces. Then a sufficient condition on the initial data is provided to ensure the occurance of the wave-breaking phenomenon. We finally prove the $\mathrm{H}^{*}$ older continuity of the data-to-solution map, and find the explicit formula of the global weak periodic peakon solution.
\end{abstract}

\section{Hosted file}

yan-qin-mu-sine-cubic-CH-v12.pdf available at https://authorea.com/users/453171/articles/ 551079-well-posedness-wave-breaking-holder-continuity-and-periodic-peakons-for-anonlocal-sine-\%C2\%B5-camassa-holm-equation 